\title{
Helmsman: fast and efficient generation of input matrices for mutation signature analysis
}

\author{
Jedidiah Carlson ${ }^{1}$, Jun Z Li ${ }^{1,2}$, Sebastian Zöllner ${ }^{3,4}$ \\ ${ }^{1}$ Department of Computational Medicine \& Bioinformatics, University of Michigan, Ann Arbor, MI, \\ USA, ${ }^{2}$ Department of Human Genetics, University of Michigan, Ann Arbor, MI, USA, \\ ${ }^{3}$ Department of Biostatistics, University of Michigan, Ann Arbor, MI, USA, ${ }^{4}$ Department of \\ Psychiatry, University of Michigan, Ann Arbor, MI, USA
}

\section{Abstract}

Motivation: The spectrum of somatic single-nucleotide variants in cancer genomes often reflects the signatures of multiple distinct mutational processes, which can provide clinically actionable insights into cancer etiology. Existing software tools for identifying and evaluating these mutational signatures do not scale to analyze large datasets containing thousands of individuals or millions of variants.

Results: We introduce Helmsman, a program designed to rapidly generate mutation spectra matrices from arbitrarily large datasets. Helmsman is up to 300 times faster than existing methods and can provide more than a 100-fold reduction in memory usage, making mutation signature analysis tractable for any collection of single nucleotide variants, no matter how large.

Availability: Helmsman is freely available for download at https://github.com/carjed/helmsman under the MIT license. Detailed documentation can be found at https://www.jedidiahcarlson.com/docs/helmsman/, and an interactive Jupyter notebook containing a guided tutorial can be accessed at https://mybinder.org/v2/gh/carjed/helmsman/master.

Contact: jedidiah@umich.edu

Supplementary information: Supplementary information for this article is available. 


\section{Introduction}

2 The spectrum of somatic single-nucleotide variants (SNVs) in cancer genomes carries important

3 information about the underlying mutation mechanisms, providing insight into the development,

4 evolution, and etiology of the cancer cell populations (Alexandrov, Nik-Zainal, Wedge, Aparicio,

5 et al., 2013). Evaluating these patterns of variation, broadly referred to as "mutational

6 signatures," has become an important task in precision oncology, as mutational signatures can

7 be used both to refine cancer diagnoses and identify effective targeted therapies (Kumar-Sinha

8 and Chinnaiyan, 2018).

9 Several software programs have been developed to aid researchers in identifying and

10 evaluating the mutational signatures present in cancer genomes (Gehring et al., 2015;

11 Rosenthal et al., 2016; Rosales et al., 2017). Most methods consider 96 mutation subtypes,

12 defined by the type of base change $(C>A, C>G, C>T, T>A, T>C, T>G)$ and the trinucleotide

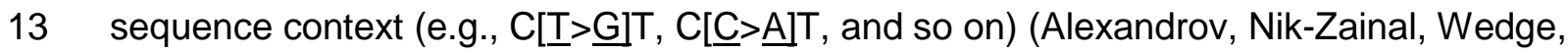

14 Campbell, et al., 2013). Mutation signature analysis methods aim to express the observed

15 mutation spectrum in each sample as a linear combination of $\mathrm{K}$ distinct mutational signatures,

16 where the signatures are inferred directly from the input data, or taken from external sources

17 such as the COSMIC mutational signature database (Alexandrov, Nik-Zainal, Wedge, Aparicio,

18 et al., 2013). These programs typically start with an input file, often in a standard format such as

19 Variant Call Format (VCF) or Mutation Annotation Format (MAF), containing the genomic

20 coordinates of each SNV and the sample(s) in which they occur. As a first step, these SNVs

21 must be summarized into a NxS mutation spectra matrix, $M$, containing the frequencies of $S$

22 different SNV subtypes in each of $\mathrm{N}$ unique samples (where the $M_{i, j}$ entry indicates the number

23 of observed SNVs of subtype $\mathrm{j}$ in sample i). Most methods are implemented as R packages and

24 must read the entire input file into memory prior to generating the mutation spectra matrix. For 
25 large input files, containing for example millions of SNVs and hundreds or thousands of

26 samples, the memory required for this step can easily exceed the physical memory capacity of

27 most servers, rendering such tools incapable of directly analyzing large datasets. To circumvent

28 these computational bottlenecks, researchers must either limit their analyses to small samples,

29 pool samples together, or develop new software to generate the mutation spectra matrix.

\section{Description}

31 To overcome the limitations of existing mutation signature analysis tools, we have developed a

32 Python application, named Helmsman, for rapidly generating mutation spectra matrices from

33 arbitrarily large datasets. Helmsman accepts both VCF and MAF files as input.

34 For each SNV in a VCF file, Helmsman identifies the mutation type based on the reference and

35 alternative alleles, then queries the corresponding reference genome for the trinucleotide

36 context of the SNV, determining subtype j. The genotypes of the $\mathrm{N}$ samples for this SNV are

37 represented as an integer array, with the number of alternative alleles per sample coded as 0,1 ,

38 or 2 according to the observed genotype (Pedersen and Quinlan, 2017). Helmsman then

39 updates the jth column of the mutation spectra matrix by vectorized addition of the genotype

40 array (i.e., $M_{i, j}$ is incremented by 1 if individual $i$ is heterozygous but does not change if

41 individual $i$ is homozygous for the reference allele). Consequently, Helmsman's processing time

42 is independent of sample size and scales linearly with the number of SNVs. The only objects

43 stored in memory are the array of $\mathrm{N}$ genotypes for the SNV being processed and the Nx96

44 mutation spectra matrix, so memory usage is independent of the number of SNVs and scales

45 linearly with sample size. 


\subsection{Additional Features}

47 In addition to being optimized for speed and low memory usage, Helmsman includes several

48 features to accommodate various usage scenarios and minimize the amount of pre-processing

49 necessary to analyze large mutation datasets. For example, if input data are spread across

50 multiple files (e.g., by different sub-samples or genomic region), Helmsman can process these

51 files in parallel and aggregate them into a single mutation spectra matrix, providing additional

52 performance improvements and avoiding the need to generate intermediate files. Similarly, in

53 certain applications, it may be desirable to pool similar samples together (e.g., by tumor type)

54 when generating the mutation spectra matrix. Helmsman can pool samples on-the-fly, without

55 needing to pre-annotate or reshape the input file with the desired grouping variable.

Helmsman also includes basic functionality for extracting mutation signatures from the mutation

57 spectra matrix using non-negative matrix factorization (NMF) or principal component analysis

58 (PCA) functions from the nimfa (Žitnik and Zupan, 2012) and scikit-learn (Pedregosa et al.,

59 2011) Python libraries, respectively. Alternatively, Helmsman can generate an R script with all

60 code necessary to load the output matrix into R and apply existing supervised and unsupervised

61 mutation signature analysis programs without requiring users to perform the computationally

62 expensive task of generating this matrix from within the $\mathrm{R}$ environment. All features are

63 described in detail in the online documentation.

\section{Results}

65 We compared Helmsman's performance to that of three published R packages:

66 SomaticSignatures (Gehring et al., 2015), deconstructSigs (Rosenthal et al., 2016), and signeR

67 (Rosales et al., 2017). We also considered several other tools and discuss their performance in

68 the Supplementary Material. For our tests, we generated a small VCF file (2.7MB compressed

69 with bgzip) containing 15,971 germline SNVs on chromosome 22 from 2,504 samples 
70 sequenced in the 1000 Genomes Project phase 3 (1000 Genomes Project Consortium et al.,

71 2015), and measured the runtime and memory usage necessary for each program to generate

72 the mutation spectra matrix. We also attempted to run each program using the full chromosome

7322 VCF file from the 1000 Genomes Project, containing 1,055,454 SNVs in 2504 individuals.

74 All programs generated the same mutation spectra matrices. Helmsman processed the small

75 VCF file in 8 seconds, with a memory footprint of $140 \mathrm{MB}$, and the full VCF file in 482 seconds

76 (linear increase for $\sim 60 x$ more variants) with no increase in memory usage as the sample size

77 remained the same. In contrast, to process the small VCF file, SomaticSignatures took 227

78 seconds with a memory footprint of $18 \mathrm{~GB}$, deconstructSigs took 2,376 seconds and $7.5 \mathrm{~GB}$ of

79 memory, and signe $R$ took 1,740 seconds and 10.2GB of memory (Fig. 1). None of these were

80 able to load the full VCF file due to memory allocation errors. All other tools we considered

81 showed similar performance bottlenecks (Supplementary Material; Supplementary Fig. S1).

82 To further highlight the speed and efficiency of Helmsman for large datasets, we evaluated the

83 entire set of $36,820,990$ autosomal biallelic SNVs from the 1000 Genomes phase 3 dataset

84 (14.4 GB when compressed with bgzip). Using 22 CPUs (one per chromosome VCF file),

85 Helmsman generated the mutation spectra matrix in 64 minutes (approximately 1.5 seconds per

86 sample), with each process requiring $<200 \mathrm{MB}$ of memory. 


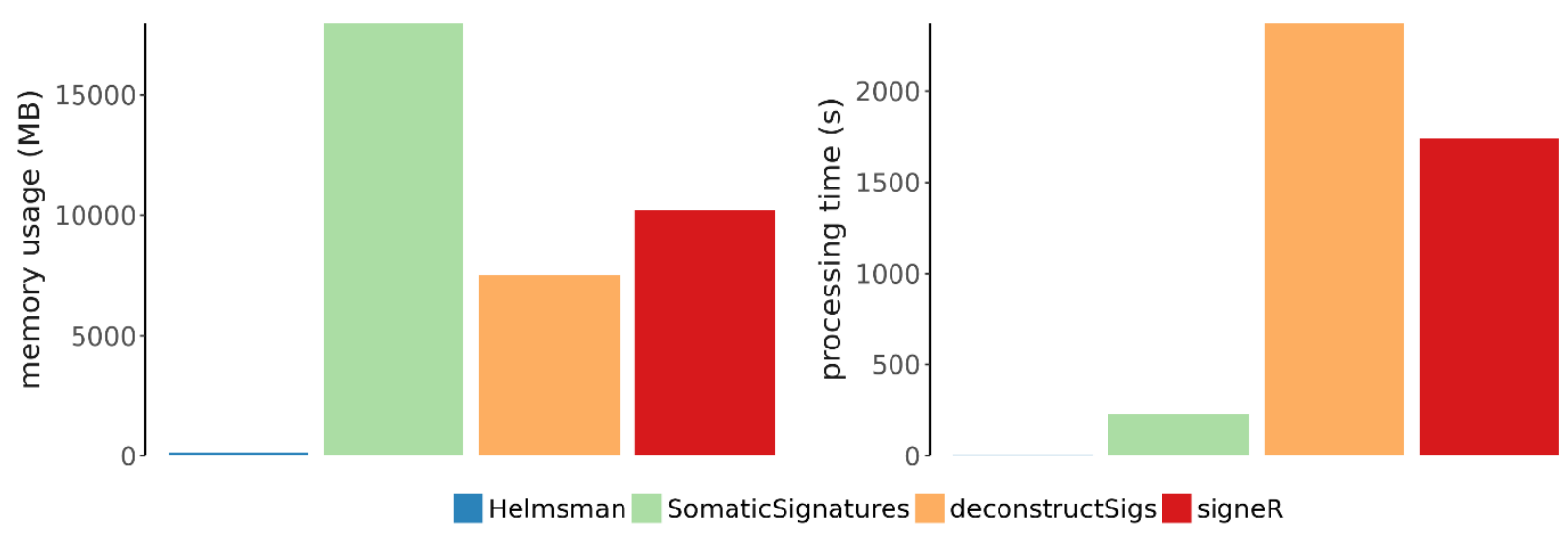

Fig. 1. Performance comparison for generation of the mutation spectra matrix from a VCF file containing 15,971 SNVs in 2,504 samples.

\section{Discussion}

88 As massive sequencing datasets become increasingly common in areas of cancer genomics

89 and precision oncology, there is a growing need for software tools that scale accordingly and

90 can be integrated into automated workflows. Our program, Helmsman, provides an efficient,

91 standardized framework for generating mutation spectra matrices from arbitrarily large, multi-

92 sample VCF or MAF files. For small datasets, Helmsman performs this task up to 300 times

93 faster than existing methods and is the only tool that can be directly applied to modern large

94 sequencing datasets.

\section{$95 \quad$ Funding}

96 This work is supported by U.S. National Institutes of Health grant R01GM118928 to JZL and SZ.

97 Conflict of Interest: none declared. 


\section{References}

99

100

101

102

103

104

105

106

107

108

109

110
1000 Genomes Project Consortium et al. (2015) A global reference for human genetic variation. Nature, 526, 68-74.

Alexandrov,L.B., Nik-Zainal,S., Wedge,D.C., Campbell,P.J., et al. (2013) Deciphering signatures of mutational processes operative in human cancer. Cell Rep., 3, 246-259.

Alexandrov,L.B., Nik-Zainal,S., Wedge,D.C., Aparicio,S.A.J.R., et al. (2013) Signatures of mutational processes in human cancer. Nature, 500, 415-421.

Gehring,J.S. et al. (2015) SomaticSignatures: inferring mutational signatures from singlenucleotide variants. Bioinformatics, 31, 3673-3675.

Kumar-Sinha,C. and Chinnaiyan,A.M. (2018) Precision oncology in the age of integrative genomics. Nat. Biotechnol., 36, 46-60.

Pedersen,B.S. and Quinlan,A.R. (2017) cyvcf2: fast, flexible variant analysis with Python. Bioinformatics.

Pedregosa,F. et al. (2011) Scikit-learn: Machine Learning in Python. J. Mach. Learn. Res., 12, 2825-2830.

Rosales,R.A. et al. (2017) signeR: an empirical Bayesian approach to mutational signature discovery. Bioinformatics, 33, 8-16.

Rosenthal,R. et al. (2016) DeconstructSigs: delineating mutational processes in single tumors distinguishes DNA repair deficiencies and patterns of carcinoma evolution. Genome Biol., $17,31$.

Žitnik,M. and Zupan,B. (2012) NIMFA : A Python Library for Nonnegative Matrix Factorization. J. Mach. Learn. Res., 13, 849-853. 